\title{
Lapurdum
}

Euskal ikerketen aldizkaria | Revue d'études basques |

Revista de estudios vascos | Basque studies review

$3 \mid 1998$

Numéro III

\section{Hendaye et ses voisines espagnoles : (1945 - années soixante) proximité géographique pour relations sporadiques}

\section{Christophe Navard}

\section{(2) OpenEdition}

1 Journals

\section{Édition électronique}

URL : https://journals.openedition.org/lapurdum/1735

DOI : 10.4000/lapurdum. 1735

ISSN : 1965-0655

Éditeur

IKER

\section{Édition imprimée}

Date de publication : 1 octobre 1998

Pagination : 299-312

ISBN : 2-84127-152-8

ISSN : $1273-3830$

\section{Référence électronique}

Christophe Navard, «Hendaye et ses voisines espagnoles : (1945 - années soixante) proximité géographique pour relations sporadiques », Lapurdum [En ligne], 3 | 1998, mis en ligne le 01 septembre 2010, consulté le 23 février 2022. URL : http://journals.openedition.org/lapurdum/1735 ; DOI : https:// doi.org/10.4000/lapurdum.1735 


\section{Christophe NAVARD}

\section{HENDAYE ET SES VOISINES ESPAGNOLES : (1945 - Années soixante) Proximité géographique pour relations sporadiques}

11 n'est pas de période plus complexe, dans les relations entre Irun, Fontarrabie et Hendaye, que celle de l'immédiat après-deuxième guerre mondiale. Alors qu'en 1945 les frontaliers s'attendaient à renouer avec leurs anciennes habitudes d'entraide mutuelle, comme ce fut le cas lors de la première guerre mondiale puis de la guerre d'Espagne ${ }^{\mathrm{I}}$, un profond blocage apparaît. Cette crise profonde, qui fait indubitablement contraste avec les périodes précédentes, limita les relations à leur plus simple aspect durant une vingtaine d'années à tel point que les logiques qui s'y retrouvent semblent être les mêmes que celles des temps passés lorsque les trois villes étaient des rivales séculaires.

Cette situation de blocage se révèle être la conséquence de plusieurs paramètres dont les racines se retrouvent aussi bien dans les faits et gestes des États concernés que dans les actions communales. Ainsi, nous pouvons déterminer trois phases successives en fonction de l'intensité des contacts. Entre 1945 et 1946, les premières hésitations et la méf iance réciproque sont telles qu'une véritable "guerre froide" se met en place. Cette phase est prolongée par une seconde qui se trouve être "l'apogée de la glaciation" des relations transfrontalières avec la fermeture de la frontière entre 1946 et 1948. Enfin, après 1948 et jusqu'au milieu des années soixante, les relations restent entachées de suspicion et d'obstacles : les communes vivent dans le spectre de la fermeture de la frontière et de ses conséquences.

\section{I - Aux temps de la méfiance réciproque (1945-01 mars 1946)}

L'orientation politique que prend la commune française est claire en 1945 : elle réfute toute notion de force, d'autorité, d'oppression et par extension tous les régimes politiques qui rappellent la violence ${ }^{2}$. De ce fait, les relations entre les trois villes restent très limitées, pour ne pas dire négligeables.

Le 19 mai 1945, "sur proposition de Monsieur Clauzet, le conseil municipal,

- constate avec douleur l'absence de deux des siens martyrisés à Dachau (Messieurs Léon Lannepouquet [ancien maire] et Artola),

- s'élève avec horreur contre la barbarie hitlérienne, et contre tous les traîtres, les dénonciateurs qui portent avec les Nazis la responsabilité de ces crimes, 
- demande que justice soit faite rapidement et s'engage à tout mettre en ouvre pour qu'il en soit rapidement ainsi,

- se réjouit en outre, de la confiance témoignée par le pays au gouvernement et au Général De Gaulle à l'occasion des élections municipales,

- souhaite que son programme de défense démocratique et réformes annoncées qui doivent permettre la Renaissance de notre Patrie, l'assainissement et la prospérité des collectivités locales soient réalisés le plus tôt possible"’3.

Suivant sa logique, au mois de juin 1945, ce même conseil s'émeut des procédés de certains services de police extérieurs à Hendaye "à l'égard d'honnêtes habitants de la commune et prône un retour à la légalité sans plus tarder"

De leur côté, les Espagnols soupçonnent leurs voisins de menées préjudiciables.

En mai 1945, une embarcation tentant de s'échapper de France est mitraillée devant Fontarrabie par un navire de surveillance militaire. Pire encore, l'exil du gouvernement basque fut longtemps à l'origine des discordes entre les deux pays. En effet, le gouvernement espagnol a toujours critiqué la situation officielle reconnue par les autorités françaises aux membres et aux délégués du gouvernement basque en exil tout en accusant ces derniers d'avoir des activités d'anarchistes sur le sol français, de préparer des attentats et des actes de sabotages qu'ils vont perpétrer en Espagne après avoir franchi la frontières.

A n'en pas douter, l'espace impliqué est un espace stratégique. La configuration géographique de la frontière reste d'ailleurs sans équivoque. Les trois villes se sont développées dans une cuvette commune où les communications restent aisées. De plus, la frontière est réduite à sa plus simple expression : une rivière, la Bidassoa, puis la baie de Chingudy séparent la France de l'Espagne. C'est dire combien, par leur extension fluviale et leur proximité elles ont été concernées par l'arrivée de réfugiés ou encore se sont senties menacées par la présence de personnes qu'elles jugeaient nuisibles ${ }^{6}$.

C'est donc dans cette région limitrophe que les exilés vont se concentrer. Les situations de Hendaye, Irun et Fontarrabie sont, en ce sens, uniques. Elles forment une sorte de promontoire qui capte toutes les pressions et les intentions de leurs régions alentours et les refoule très facilement vers l'adversaire.

Par conséquent, c'est face à ce phénomène que les populations se trouvent confrontées jusqu'en 1946. Ensuite, elles composent avec une mesure diplomatique beaucoup plus délicate et restrictive : la fermeture de la frontière.

\section{II - La fermeture de la frontière (01 mars 1946-10 février 1948).}

En application de la décision du gouvernement socialiste de Félix Gouin, la frontière franco-espagnole est fermée à toute circulation de personnes et de marchandises dès le $1^{\text {er }}$ mars 1946 et reste effective jusqu'au 10 février 1948.

Les trois villes héritent des principales conséquences de cette mesure qui est localisée sur la frontière. 
Aussi bien les économies locales que les liens entre familles mixtes se retrouvent anéantis et la crise engendrée par cette décision ne fait que contribuer à l'échec de leurs relations déjà très exiguës.

\section{1-Les mesures ministérielles et leurs répercussions locales.}

Les mesures d'application examinées par chaque département ministériel français à la suite des deux réunions des 26 et 27 février 1946 aboutissent à l'isolement des frontaliers et au déclin économique.

\section{a) Isolement des hommes et des familles.}

Les séparations provoquées par la décision sont effroyables et ne laissent aucun doute sur les conséquences désastreuses qu'elles ont engendrées. L'isolement des habitants se perçoit à la lecture des dispositions des Ministères de l'Intérieur, des PTT et de la Défense Nationale :

Sur décision du Ministère de l'Intérieur, aucun franchissement n'est toléré hormis celui des personnes accréditées:

"La fermeture en ce qui concerne le trafic terrestre, maritime et aérien s'applique à toutes les personnes, quelle que soit leur nationalité à l'exception des personnes diplomatiques et consulaires, du courrier diplomatique, des personnes de l'UNRRA et de la Croix Rouge Internationale, des ressortissants portugais regagnant leur pays".

De même, les clauses concernant les frontaliers sont draconiennes et les familles mixtes voulant voir leurs proches en sont empêchées :

"La fermeture ne fait pas obstacle aux Français vivant en Espagne et voulant regagner leur pays. Par contre, les Espagnols établis en France ne peuvent regagner l'Espagne sauf si leur gouvernement émet des conditions au retour des Français".

Plus graves encore, l'isolement physique imposé officiellement est relayé par un éloignement moral puisque le Ministère des PTT :

“'A. interdit les échanges entre la France et ses possessions et l'Espagne et ses possessions,

B. suspend les correspondances télégraphiques privées et maintient la non reprise des communications téléphoniques".

Les voisins basques se retrouvent sans aucun contact physique, acoustique ou écrit. Ils en sont réduits à vivoter sans pouvoir se tourner vers l'un des débouchés natureis dont ils disposaient auparavant et qui leur fournissait de quoi mieux vivre. Qui plus est, ils se sentent bafoués ${ }^{8}$ puisqu'ils ont l'impression d'être privés d'un droit fondamental qu'ils détenaient auparavant.

Face à cette situation, beaucoup souhaitent se tourner vers l'illégalité mais doivent faire face à la répression de plus en plus présente car "le Ministère de la Défense Nationale s'efforce de fournir les effectifs supplémentaires nécessaires au contrôle de la frontière des Pyrénée.:". 
Si le désagrément atteint son comble sur le plan moral et humain, la fermeture de la frontière va provoquer un sinistre économique réel et des frustrations encore plus importantes.

\section{b) Un désastre économique.}

La suite de l'analyse de la note révèle que les répercussions sont aussi d'ordre économique.

"Le Ministère des Travaux Publics et des Transports décrète:

A. une interruption du transport d'origine française

B. une interruption du transport d'origine espagnole, par fer et route,

C. l'interdiction des bateaux espagnols dans les ports français,

le vol et le survol du territoire français, les escales,

D. I'interdiction du transit des marchandises à destination ou en provenance de la Suisse, exception faite de celles du Portugal et au-delà".

Complétée par les décisions du Ministère de l'Économie et des Finances qui stipulent "que l'entrée des marchandises espagnoles et la sortie des marchandises françaises sont interdites; cette mesure n'étant pas applicable pour les marchandises de/vers le Portugal", l'ampleur des dommages est considérable.

Tout mouvement de marchandises relevant du simple échange est prohibé à l'échelle locale. Seul le transit des marchandises à destination du Portugal est autorisé, ce qui constitue une maigre consolation dans la mesure où les activités fer de lance des villes en sont réduites à leurs plus simples intérêts voire à néant.

\section{2 - A mesures drastiques, conséquences pernicieuses.}

a) Une économie amputée.

- Développement du chômage.

Le chômage qui jusqu'alors touchait très peu les villes, compte tenu de leurs activités à vocation transfrontalière, se développe de manière régulière au point d'effrayer une large partie de la population.

La population hendayaise est de plus en plus inquiète et formule plusieurs vœux pour la réouverture de la frontière'. En effet, elle est la plus touchée par la crise. Lorsque la fermeture de la frontière est officielle, c'est la pleine saison des agrumes, or "ce trafic, qui se produit précisément pendant la période creuse de l'hiver, contribue pour une bonne part, à la prospérité laborieuse de Hendaye"'10.

Plus grave encore, la population active des trois villes est menacée. "Les marchandises à l'importation sont soumises à d'importantes opérations de manutention : le transbordement direct de wagons espagnols sur wagons français ou le déchargement de wagons espagnols à quai puis le chargement du quai dans les wagons français emploie une main d'œuvre considérable"'!. Et, c'est cette dernière, hendayaise ou espagnole, qui se trouve privée de son emploi temporaire, qui bien souvent lui permettait de vivre une partie de l'année, et en concurrence, 
puisque le travail n'était pas assez substantiel bien que les Espagnols n'aient plus le droit de franchir la Bidassoa.

\section{- La désertion espagnole.}

Depuis de longues années, Français et Espagnols se rendaient librement dans le pays opposé. Les Espagnols, eux, fréquentaient l'ensemble de la côte basque de Biarritz à Hendaye et jouaient dans les casinos. C'est donc ce manque à gagner qui va faire souci à l'échelle des communes françaises.

Alors qu'elle est saisie d'une demande en vue d'autoriser la reprise de l'exploitation du casino de la part de la Société Anonyme de la Baie de Saint-Jeande-Luz, propriétaire de l'immeuble de style mauresque ${ }^{12}$, la municipalité espère pouvoir tirer bénéfice de son exploitation et propose un cahier des charges qui fait état d'une taxe perçue d'un montant de 200000 francs ( 100000 pour la participation aux fêtes locales, 100000 pour la publicité en général). Mais dès le $23 \mathrm{du}$ même mois, elle le revoit à la baisse car "la saison ne sera pas aussi bonne qu'espérée, la situation de la ville étant problématique à cause du maintien de la zone interdite qui constitue un obstacle à l'activité saisonnière et de la fermeture de la frontière, qui empêche la venue des touristes espagnols"'s.

Le maintien de la réglementation existante, le statut de zone interdite, placent les villes frontalières françaises dans une situation particulièrement embarrassante et constitue une entrave certaine à l'essor économique et touristique de cette région ${ }^{14}$. De plus, elle provoque à la longue, une crise morale majeure.

\section{b) Une crise des valeurs profonde.}

Ce marasme se perçoit grâce à une situation humaine malsaine et aux rivalités omniprésentes.

- Une situation alambiquée.

Hendaye accueille pêle-mêle des réfugiés politiques et des individus vivant en Espagne et qui ne supportaient plus d'être séparés de leurs familles, les premiers ne voulant pas retourner en Espagne et les second ayant l'espoir d'y revenir un jour. Toutefois, le vœu pour la réouverture de la frontière signale que "sur le plan humain, cette situation interdit à de nombreux foyers franco-espagnols d'entretenir des relations que réclament légitimement les liens qui les unissent" ${ }^{15}$.

Si l'on se réfère aux résultats du recensement de la population de 1946 et aux études du Quai d'Orsay, Hendaye héberge 961 personnes étrangères sur 6251 habitants; selon le dépouillement précis réalisé à la Mairie, une proportion de 94 à $95 \%$ de cette population est espagnole. Ainsi, l'immigration hendayaise est avant tout espagnole.

Ces chiffres reflètent un fait sociologique capital : de nombreux foyers sont issus de familles mixtes ${ }^{16}$; ainsi, bien des individus n'ont plus aucune relation avec leurs parents espagnols et vice-versa, du moins officiellement.

Enfin, ils peuvent aussi nous renseigner sur un phénomène politique et économique plus large : la venue de réfugiés espagnols lorsqu'éclate la guerre d'Espagne et au moment de l'incendie d'Irun le 2 septembre 1936, puis pendant la seconde guerre mondiale. Leur nombre exact n'est pas connu compte tenu de 
la situation troublée qui secouait la ville et de leur déplacement. Certains avancent le chiffre de près de 20000 personnes accueillies ${ }^{17}$; des renseignements plausibles et très précieux ont pu être obtenus auprès du Maire de l'époque par Raymond Lecuyer, envoyé spécial de la revue L'Illustration: "entre le 31 août et le 10 septembre 1936,13510 repas quotidiens ont été servis gratuitement. Pendant quelques jours, 9428 réfugiés ont été hébergés à Hendaye, auxquels s'ajoutent 4000 immigrés dont la situation n'était pas facile à dresser puisqu'ils étaient logés chez l'habitant à titre onéreux ou gratuit.

Par la suite, ils ont été dirigés vers d'autres villes de France : dans le département à Cambo, Ciboure, Bayonne, Sare, Anglet"18 car l'administration cherchait à les éparpiller partout. Seuls restaient ceux qui pouvaient justifier avoir de la famille en ville, ceux qui avaient les moyens de se financer un séjour. Les autres repartaient vers l'Espagne par la frontière de Dancharia et revenaient vers Irun, par la frontière catalane et s'installaient alors en zone libre dans la région de Barcelone.

\section{- Des rivalités exacerbées.}

La crise économique favorise les incompréhensions de part et d'autre de la frontière. Il suffit pour s'en rendre compte de prendre un exemple apparemment aussi insignifiant que le programme de protection de la plage. "Les travaux ont été menés à bien jusque fin 1947 et depuis, suite au manque de crédits mis à la disposition par le Ministère des Travaux Publics, aucune transformation n'a été réalisée" 19 . La ville confrontée aux dures réalités de la crise économique locale voit son projet de modification des travaux reporté alors que du côté espagnol, ils se poursuivent. Cette situation équivoque est mal comprise : comment, dans un pays dont l'économie est encore plus touchée par la récession, une ville arrive-telle à bâtir sa digue sans manifester plus de gêne ? Malgré toutes les difficultés éprouvées ${ }^{20}$, les Ondarribitars continuent à canaliser le lit de la Bidassoa et donc à préserver leur ville alors que les Français, eux, demeurent incapables de financer les travaux et sont contraints d'attendre. Hendaye se retrouve privée de sa faculté naturelle la plus charmeuse : sa longue plage de sable blanc et fin est négligée et se détériore, à l'image du mur de soutien de la R.N.10 C qui s'affaisse, des routes et trottoirs qui, seulement "rafistolés", restent défoncés.

\section{III - La réouverture de la frontière : une tentative avortée pour des logiques conservées (10 février 1948-années soixante).}

A l'heure de la réouverture de la frontière une dialectique semble l'emporter : celle de la fermeture. Bien que les actions en vue d'une nouvelle situation avec l'Espagne soient nombreuses, la crainte manifestée lors d'une réunion du conseil municipal de Biarritz semble se confirmer : si un pas a été franchi, l'isolement prime $^{21}$. La désorganisation et la déconcertation qui découlent de cet état de fait sont flagrantes. Pratiquement aucune relation directe n'est relevée jusqu'en 1957.

\section{I- Un déblocage progressif.}

La réouverture de la frontière s'effectue en plusieurs étapes et non pas seulement le 10 février 1948. En effet : 
- depuis 1946 les dérogations et assouplissements ayant trait au passage de la frontière se sont succédés; on dénombre au Quai d'Orsay au moins dix documents qui réclament des mesures de clémence entre les 6 mars et $1^{\text {er }}$ septembre 1946. Cette dernière est importante concernant la vie frontalière dans la mesure où elle assouplit les normes de circulation pour les personnes pouvant faire état d'impératifs professionnels ou familiaux.

- la frontière n'est pas totalement rouverte le 10 février 1948 : seuls le passage des personnes et les communications par voie postale, téléphonique et télégraphique sont autorisés. Ce n'est que sous délai de quinze jours" à un mois ${ }^{23}$, que le rétablissement du transit marchandise est envisagé, notamment à destination de la Suisse.

Enfin, la reprise des échanges, entérinée par de nouveaux accords commerciaux entre les deux pays, "reprendra ses droits ultérieurement vu que l'accord de Saint-Sébastien précisant le volume des échanges est dès à présent inadapté"

\section{2- Des rapports très parcimonieux.}

\section{a) Reprise de l'aide alimentaire.}

La pénurie drastique qui touche à la fois la France et l'Espagne est une crise caractéristique d'après guerre. En effet, rien n'a évolué depuis 1945 : les insuffisances du ravitaillement tant en quantité de denrées rationnées que dans le fonctionnement du service départemental se font rudement ressentir. La ville va donc essayer, compte tenu de sa position géographique, de régler ce manque en se tournant vers l'Espagne comme elle l'avait fait en $1945^{25}$. Si elle pouvait le faire concernant certains produits spécifiques comme les conserves de poisson, l'huile d'olive, la farine, les pommes de terre, les bananes et les citrons - surtout réservés à l'exportation - ce sont particulièrement les voisines espagnoles qui recourent à l'aide française ${ }^{26}$.

La situation en Espagne est des plus préoccupantes et contraint le gouvernement à diminuer les rations alimentaires (moins 50 grammes de pain par jour) ${ }^{27}$. A côté de ceci, "le coût de la vie ne cesse de croître [et] les salaires ne peuvent plus suivre la hausse des prix de toutes les marchandises (...) Chacun s'arrange comme il peut : ainsi font les frontaliers qui passent chaque jour en deux files le pont de Hendaye, les Espagnols venant chercher le pain en France, les Français le vin en Espagne... Le Basque reçoit :

- 200 grammes de sucre par jour,

- un quart de litre d'huile,

- 200 grammes de mauvais pain par jour ( 350 pour les travailleurs de force)

- aucune viande" 28 .

Cette situation, et celle exposée dans le tableau ci-après, expliquent l'aide apportée par Hendaye aux Irunais et Ondarribiars. Bien que reflétant les conditions d'échange de 1950, les Espagnols, compte tenu du taux de change des monnaies en vigueur jusqu'à la fin des années cinquante n'avaient que des avantages à venir s'approvisionner en France ${ }^{29}$. 


\section{PRIX COMPARES DES BIENS DE CONSOMMATION. \\ (1950)}

\begin{tabular}{|c|c|c|c|c|c|}
\hline & \multicolumn{2}{|c|}{ EN ESPAGNE } & \multicolumn{2}{|c|}{ EN FRANCE } \\
\hline & & PESETAS & FRANCS & PESETAS & FRANCS \\
\hline \multicolumn{6}{|c|}{ VIANDES } \\
\hline Mouton & $\begin{array}{l}1 \mathrm{~kg} \\
1 \mathrm{~kg}\end{array}$ & $\begin{array}{l}50.00 \\
4500\end{array}$ & $\begin{array}{l}343.00 \\
490.50\end{array}$ & 0.23 & $\begin{array}{l}2.19 \\
3.63\end{array}$ \\
\hline Porc & $1 \mathrm{~kg}$ & 65.00 & 708.50 & 0.19 & 2.06 \\
\hline \multicolumn{6}{|c|}{ FRUITS/LÉGUMES } \\
\hline Oranges & $1 \mathrm{~kg}$ & 6.00 & 65.40 & & \\
\hline P. de terre & $1 \mathrm{~kg}$ & 6.50 & 70.85 & 0.07 & 029 \\
\hline \multicolumn{6}{|c|}{ MAT. GRASSES } \\
\hline Beurre & $1 \mathrm{~kg}$ & 5.00 & 708.50 & 0.63 & 6.91 \\
\hline Huile & 1 litre & 35.00 & 381.50 & 0.29 & 3.18 \\
\hline \multicolumn{6}{|c|}{ AUTRES DENRÉES } \\
\hline Café & $1 \mathrm{~kg}$ & 100.00 & 1090.00 & - & - \\
\hline Sucre & $1 \mathrm{~kg}$ & 28.00 & 305.20 & 0.06 & 0.63 \\
\hline Lait & 1 litre & 6.00 & 65.40 & - & - \\
\hline Vin & 1 litre & 7.90 & 86.11 & 0.07 & 0.77 \\
\hline \multicolumn{6}{|c|}{ AUTRES BIENS } \\
\hline Charbon & $1 \mathrm{t}$. & 750.00 & 8175.00 & - & - \\
\hline Complet $\mathrm{h}$ & & 1600.00 & 1740.00 & - & - \\
\hline
\end{tabular}

SOURCE : A.M.A.E., dossier 130 , note 32 EU.

J. Fourastic et B. Bazil : Pourquoi les prix baissent?

Elaboration personnelle.

Si cette aide précieuse est appréciée aussi bien des uns que des autres, il ne faudrait pas croire qu'elle va contribuer à un rapprochement durable. En effet, l'échange alimentaire est la seule forme de relation entre habitants du bassin de la Bidassoa. Au-delà, le terrain cède plus volontiers la place aux crispations et aux irritations.

b) La présence des réfugiés : un poison pour les relations transfrontalières officielles.

La présence des réfugiés politiques à Hendaye a toujours été source de problème au vu des autorités de Madrid. Pourtant, selon une note du Ministère des Affaires Étrangères, ils sont nombreux et restent largement concentrés sur une quinzaine de kilomètres aux alentours de la frontière ${ }^{30}$ :

"6\% des ressortissants espagnols sont soldats et désertent l'armée pour motif réellement politique, 
$5 \%$ sont d'anciens prisonniers des camps de concentration ou des prisons franquistes revenus dans leurs familles et craignant de nouvelles représailles,

$5 \%$ sont d'anciens fonctionnaires et intellectuels qui ne peuvent plus exercer leur profession en Espagne par interaction politique".

A leur côté, des membres du gouvernement basque en exil sont source de tiraillement.

Lors d'un communiqué du $1^{\text {er }}$ mai 1951 , sur 15 noms de dirigeants basques soupçonnés d'être les plus actifs dans la région du Sud-Ouest, plus de la moitié intéresse notre champ d'étude :

- trois sont de Hendaye, le directeur des activités du Bureau central de Paris, le chef de service de la Délégation dont l'importance est très grande pour l'organisation clandestine, l'ex-chef de la brigade du Bas-quartier de Hendaye,

- cinq sont de Saint-Jean-de-Luz : le Président du gouvernement, le directeur du Bureau de "Résistance basque", le directeur du Centre des Activités basques, le Conseiller du gouvernement et le directeur de "Radio Euskadi".

Ainsi, l'ensemble des archives du Ministère des Affaires Étrangères ne fait que se reporter à cette situation. Au niveau national, un phénomène de cristallisation freine toute relation. Les archives municipales, quant à elles, ne font que renseigner sur une autre difficulté : le passage de la frontière.

\section{c) Le problème du franchissement de la frontière.}

Ce problème est crucial au lendemain de la réouverture de la frontière. Alors qu'il "semble réglé" à l'échelie nationale, du moins théoriquement, un blocage naît à Hendaye. Dès le 7 avril 1948, neufs conseillers municipaux rédigent une lettre à l'attention de Monsieur le Maire, constatant que "si la frontière est rouverte, nos populations par contre n'ont pas encore le plaisir de franchir les barrières" ${ }^{31}$. Ce fait est, en fait, attribuable à plusieurs explications :

- Le visa.

L'obtention du visa nécessaire, tant par son prix que par le délais d'attente, auprès des autorités françaises est très difficile.

Le prix de la redevance pour un visa valable une seule journée est exagéré, ce qui place la plupart des ressortissants français dans l'impossibilité d'obtenir les devises exigées par les autorités espagnoles pour un voyage de longue durée. Ils confient donc au Maire "le soin d'intervenir auprès de qui de droit pour obtenir du gouvernement le retour aux facilités qui étaient accordées à tous les frontaliers avant $1935^{\prime \prime 32}$. Cette question du prix reste latente et les Hendayais n'ont de cesse de dénoncer le régime de faveur de certains puisque "seule une catégorie de la population bénéficie de facilités de passage alors que beaucoup de familles n'obtiennent même pas un visa" ${ }^{33}$.

De nouveau, en 1949, la question du délai est reposée $e^{34}$ et en 1954, c'est au tour de celle de son prix: "Monsieur Bienabe signale que le prix des visas vient d'être augmenté alors que chacun souhaite un assouplissement réel des conditions de passage de la frontière. Par la même occasion, et une nouvelle fois, le conseil 
municipal charge le premier élu d'intervenir auprès des autorités françaises pour «qu'elles proposent à nos voisins la suppression des visas »"3.

Il s'engage, à partir de cet instant, toute une discussion à propos de ces visas et des laissez-passer : quels rôles peuvent-ils avoir alors que les modalités de passage sont plus draconiennes que jamais? Ils ne contribuent pas à assouplir les conditions de franchissement mais sont plutôt représentatifs d'une régression puisqu'ils datent, à l'origine, d'une époque où la frontière n'était qu'entrouverte et où seuls quelques privilégiés (agents en douane, fonctionnaires, en activité et en retraite et leurs familles) étaient favorisés. Maintenant qu'elle est accessible à tous, ils n'ont toujours pas disparus et demeurent donc source d'inégalité ${ }^{36}$. Selon cette même source, "la délivrance des laissez-passer est la résultante de conventions réciproques entre les services de Police des deux côtés de la frontière (...) et ce droit de passage gratuit ne devrait pas être l'apanage d'une partie de la population au détriment de l'autre". Tous doivent avoir les mêmes devoirs mais aussi les mêmes droits.

Ainsi, les frontaliers français proposent des solutions - suppression totale des laissez-passer actuels pour les remplacer par des laissez-passer valables pour un membre d'une famille; les autres étant astreints aux passeports et visas - et des tentatives d'assouplissement des formalités mais qui jamais n'aboutissent.

Au contraire, la totale désorganisation qui s'ensuit va engendrer une rivalité accrue et le franchissement de la frontière n'en sera que plus difficile.

\section{- La désorganisation de la vie frontalière.}

Dès l'année 1950, de nombreuses requêtes arrivent à la Mairie et rapportent une situation désorganisée de laquelle les voisins espagnols sortent grands vainqueurs. La ville doit résoudre plusieurs problèmes dont le principal est l'invasion constante du marché du travail français par une masse laborieuse espagnole.

\section{L'inégalité laborieuse.}

L'inégalité du travail tend progressivement à s'étendre entre les frontaliers. A tout instant, les archives révèlent une situation préjudiciable au sujet des activités de transit aussi bien des marchandises que des personnes.

Lors de la réunion du 22 décembre 1950 un conseiller demande "à ce que les transbordeurs d'oranges utilisent en priorité la main-d'œuvre française, alors que le recours à celle venant journellement d'Espagne est de plus en plus fréquent". Seulement cette activité est soumise à une réglementation sans cesse mouvante ce qui ne facilite pas la tâche des dits transbordeurs ${ }^{37}$ et ravive les tensions : 1'embauche d'une main-d'œuvre française nombreuse est en concurrence avec la pénétration espagnole.

Dans cette optique : le cas de l'invasion de plus en plus conséquente "des taxis, de Saint-Sébastien qui conduisent à l'intérieur de notre pays des touristes ou des gens que leurs affaires poussent chez nous" 38 est craint. Tout comme les oranges, les taxis français sont en concurrence avec les taxis espagnols puisqu'ils ne bénéficient pas de la réciprocité légitime qu'ils réclament - "nous ne demandons pas à ce que l'on empêche les taxis espagnols de venir en France, mais très 
énergiquement à bénéficier, à notre tour, de l'autorisation de conduire nos clients éventuels à toutes destinations en Espagne" - ce qui est très préjudiciable car il leur est interdit de passer le pont international d'une part et qu'ils perdent de nombreuses courses sur le territoire français alors que leurs homologues espagnols possèdent ce privilège. Toutefois, selon le Traité du travail conclu en 1932, l'assimilation des Français aux Espagnols était prévue en ce qui concerne le régime du travail dans la Péninsule, mais les ressortissants français étaient assujettis, pour obtenir une carte de travail, à acquitter une taxe beaucoup plus lourde que celle prévue pour les nationaux.

Une question se pose donc : les Français peuvent-ils se permettre d'aller travailler en Espagne tout comme le font les Espagnols? La réponse semble être négative tant les rigidités sont lourdes et nombreuses.

\section{L'attitude des douaniers.}

L'attitude des douaniers envers "les Espagnols, qui pour la plupart sont de modestes travailleurs venant faire leurs achats en France pour pouvoir alimenter un peu mieux leurs nombreuses familles, avec leurs petits traitements et salaires inqualifiables" parait déconcertante ${ }^{39}$. Ainsi, la plupart des Hendayais s'indignent des brimades infligées à leurs camarades espagnols ${ }^{40}$ et réagissent vigoureusement : "cette attitude n'est pas comparable à celle de leurs collègues [espagnols], qui leur permettent de se procurer à bon compte tout ce dont ils ont besoin, non seulement en produits alimentaires mais encore en chaussures, habillement....".

\section{L'instauration du sens unique de passage.}

L'instauration d'un sens unique de passage de la frontière mécontente la majeure partie de la population et suscite l'intervention du Préfet des BassesPyrénées.

C'est pour désengorger le pont international de Hendaye qu'une réunion de nombreuses personnalités, de hauts fonctionnaires français et espagnols représentant les divers ministères a lieu sans consultation préalable des membres et organismes locaux les plus concernés semble-t-il : Maire, syndicat d'initiative, adhérent du tourisme local... Par une lettre de protestation expédiée de l'office du tourisme le 17 mars 1951, le lecteur apprend que depuis deux ans l'affáre du sens unique à la frontière ne cesse d'être à l'ordre du jour.

Ce sens unique, est à nouveau, pour Hendaye synonyme de ruine économique - une des conséquences est d'éloigner la masse touristique - et d'entrave aux bonnes relations entre voisins "si comme envisagé, la rentrée en Espagne s'effectue par Hendaye et la sortie par Béhobie". Les frontaliers voulant venir en France ou se rendre en Espagne sont tributaires d'un parcours imposé qui pour certains les obligent à faire un détour conséquent.

Ainsi une mobilisation générale se met en place en vue de retrouver la liberté et le choix de passage - "s'il y a embouteillage, la faute en revient aux ditficultés déjà connues (formalités exigées par les polices, douanes, change...) " et de rassurer les voisins "d'outre-Pyrénées" ", hormis la voix d'un conseiller municipal 
qui se joint aux protestations mais déclare voter contre le passage relatif aux relations amicales avec l'Espagne".

Cependant rien n'y fait. En 1952, un nouveau vœu du conseil municipal dénonce, devant les protestations des frontaliers, "l'obligation faite aux usagers hendayais de n'emprunter qu'une seule route de bureau et insiste pour que la mesure en vigueur soit reconsidérée par l'Administration des douanes en vue de donner à la dite tolérance une forme plus rationnelle et aussi de permettre à tous le retour en France par les voies qui conviennent le mieux à chacun" ${ }^{\prime 3}$.

La ville subit de nouvelles mesures restrictives qui peuvent s'avérer dangereuses pour sa survie. Pourtant, les élus ne renoncent pas malgré ces blocages successifs à poursuivre leur politique d'entraide amorcée quelques années auparavant.

A l'attirance naturelle et historique dont il était logique de retrouver trace se substituent des relations exiguës et complexes. Oscillant entre nouveaux contacts ou porte close les relations transfrontalières s'enfoncent dans la confusion, cette dernière étant largement alimentée par la défiance réciproque des États.

Rien n'est à même de recréer la coopération d'antan : peur du franquisme et de l'autre, fermeture de la frontière, deux ingrédients qui cèdent la place aux relations de contiguïté et non à la volonté de renouer des liens. Les seuls contacts ont leur racine dans le fait même du voisinage ${ }^{44}$.

Cette situation perdure jusqu'au milieu des années soixante, moment où apparaissent les éléments d'une nouvelle donne internationale. C'est sous l'effet des assouplissements du régime franquiste et de la croissance économique au niveau national, puis de l'apparition d'institutions et de manifestations culturelles diverses comme le journal Hendaye-Echos, les commémorations de la mort de Pierre Loti ou du centenaire du chemin de fer que les villes voient s'ouvrir d'autres perspectives.

\section{Christophe NAVARD}

Université de Paris I - Panthéon - Sorbonne

Centre Pierre Renouvin 


\section{Notes}

1 - Pour de plus amples renseignements se reporter à notre travail d'études et de recherches: Hendaye entre fermeture et owverture (1945-1975), chapitre I "La Bidassoa, une frontière ou un gué ?, pp. 29-35. Mémoire de maîtrise d'histoire des relations franco-espagnoles sous la direction de René Girault, Université Paris I, 1994.

2 - "elle repousse systématiquement ce qui rappelle le totalitarisme allemand et donc ce qui lui a été affilié, même l'Espagne franquiste", in C. Navard, op. cit., chap. 2 "Une vie internationale limitée : la crise d'après-guerre et le fermeture de la frontière (1945-1948)", p.48.

3 - Délibération du Conseil Municipal n 45/296, 19 mai 1945, Mairie.

4 - Id., $n^{\circ} 45 / 285,30$ juin 1945.

5 - Archives du Ministère des Affaires Étrangères de Paris (ci-après A.M.A.E), Note de la Direction Europe pour le Ministre des Affaires Étrangènes du 18 août 1949, dossier 154.

6 - “il est probable que d'autres arrivées ont eu lieu hors de contrôle, les plages étant sans surveillance", ibidem.

7 - A.M.A.E., Dossier 84, Note d'information du 28 février 1946.

8 - Vou pour la réouverture de la frontière, Délibération $n^{\circ} 47 / 596,28$ novembre 1947.

9 - En tout trois vœux ont été formulés: les $18 / 9$ février 1946 au sujet de la zone interdite, 01 septembre I 946 et 28 novembre 1947.

10 - Chambre de Commerce de Bayonne, "Le rôle de Hendaye dans les relations économiques de l'Espagne avec les autres pays européens" dans Activité en Pavs basque n"48 spécial Hendaye, février 1954.

II - Ibidem.

12 - Chambre de Commerce de Bayonne, "Note d'étude sur la fermeture de la frontière et sess conséquences".

13 - Chambre de Commerce de Bayonne, idem

14 - A.M.A.E, lettre de l'Assemblée nationale constituante au Quai d'Orsay des 18/9 février 1946 , dossier 84 , Série Z.

15 - Vcu pour la réouverture de la frontière, op. cit.

16 - Certains s'avancent mème à prétendre que chaque Hendayais a parmi les membres de sa famille au moins un Espagnol vivant à Irun ou Fontarrabie.

17 - Abbé Michelena, Histoire d'Hendaye, T.1, Hendaye : son histoire. Hendaye, Haize Garbia, 1987, p. 621 .

18 - Article de presse de Raymond Lecuyer cité par Abbé M. Michelena, op. cit., p. 623.

19 - Registre des délibérations du conseil municipal, $\mathrm{n}^{\circ} 48 / 702,12$ novembre I948. Il faut ajouter qu'à l'origine le programme de protection de la plage était dû à l'initiative de Fontarrabie puisque travailler sur le cour d'eau international nécessitait d'avoir l'accord des deux parties. En ce sens, il contribua à un rapprochement.

20 - "beaucoup de travail, faibles revenus, penurie généralisée [même si] le Pays basque espagnol, région industrielle, est moins touché par la crise du moins jusqu'en 1950" in Bartolomé Bennassar, Histoire des Espagnols, Livre 2, Chapitre 10 " La post guerre : le travail et l'austérité ".

21 - A.M.A.E, Lettre du Préfet des Basses-Pyrénées au Ministre des Affaires Étrangères du 30 janvier 1948 au sujet de l'ouverture de la frontière, dossier 86.

22 - Journal Le Monde, 08/09 février 1948. 
23 - A.M.A.E, Télégramme au départ du Ministère des Affaires Étrangères pour le Palais de Santa Cruz du 22 janvier 1948 au sujet des étapes de la réouverture de la frontière, dossier 86

24 - Ibidem

25 - "Des le 30 juin 1945, Monsieur le Maire de Hendaye divulgue l'accord de l'Administration des Douanes à pouvoir importer des oranges afin de l $\cdots$ répartir à la population”, Délibération $n^{\circ} 45 / 274$, Registre Mairie.

26 - A.M.A.E., dossier 154, note du 01 juin 1945.

27 - B. Bennassar, op. cit., chap. 10.

28 - A.M.A.E., dossier 130, note $n^{\circ} 32$ EU du Consulat français de Saint-Sébastien au Mínistère des Affaires Étrangères, Série Z

29 - Selon le taux de change en vigueur $-4,56 \mathrm{~F}$ la peseta en 1945 et 1946, 10,85 à 10,95F entre 1947 et 1956 et 8,19 à $8,20 \mathrm{~F}$ entre 1957 et 1959 - les prix en France étaient 100 à 800 fois moins chers, in J.M. Delaunay, Des Palais en Espagne : l'École des Hautes Études et la Casa de Velazquez au cour des relations franco-espagnoles du XX' siècle (1909-1979), Thèse de $3^{\circ}$ cycle, Paris I, 1988.

30 - A.M.A.E, Note des Renseignements généraux... au sujet des Espagnols franchissant la frontière clandestinement du II mai 1946, op. cit.

31 - Lettre des conseillers municipaux au Maire de Hendaye, Documents annexes aux délibérations du conseil municipal, Archives municipales, Série D l d.

32 - Délibération $n^{\circ} 48 / 643$ du conseil municipal du 08 avril 1948.

33 - Idem, $\mathrm{n}^{\circ} 49 / 761$.

34 - lbidem.

35 - Id., n54/428.

36 - "il a toujours existé depuis la Liberation, à Hendayc, deux sortes de frontaliers : ceux qui payent el ceux qui franchissent la frontière gratuitement", Lettre de Monsieur Lhosmos, retraité SNCF en date du 16 novembre 1950, Doléances et correspondances, Archives municipales, Série D 2 b..

37 - Télégramme de Monsieur de Chevigné-Chaze (Assemblée Nationale) à Monsieur Guy Petit de mars 1951 qui nous apprend que « 1000 tonnes d'agrumes sont bloquées en gare de Hendaye par suite de l'application du rectificatif du contingent autorisé à l'importation par Hendaye fixé le 20 février à 6000 tonnes, ramené à 3000 tonnes par le $J . O$. numéro 15 en mars 1951, Télégramme d' information..., Courriers, correspondances et doléances, Archives municipales, Série D 2 b.

38 - Lettre du groupement des taxis de la ville de Hendaye au Maire, Courriers, correspondances et doléances, Archives municipales, Série D 2 b.

39 - Lettre de Monsieur José Garay, du 11 janvier 1950, Archives municipales, série D 2 b

40 - « il ne se trouve pas un seul homme parmi eux, qui pourra me prouver que du côté espagnol on ne leur aie pas toujours donné toutes sortes de facilités pour passer tout ce qui leur plait m, ibid.

41 - "les relations amicales des deux pays ne pourront se développer que par les facilités accordées pour le passage de la frontière".

42 - Délibération du conseil municipal n 51/37, 27 février 1951.

43 - Délibération du conseil municipal, 18 mars 1952.

44 - Carlos Fernandez de Casadevante Romani, La frontière franco-espagnole et les relations de voisinage avec une référence spéciale au secteur du Pays basque, Harriet, Bayonne, 1989, $1^{r a}$ partie, chapitre I "les relations de voisinage : aspects généraux", p. 34. 